\title{
AN ENQUIRY INTO THE VALUE OF AUTOHAEMOTHERAPY IN JUVENILE ASTHMA
}

BY

\author{
KEMPSON MADDOX, M.D. (SydNey), M.R.C.P.,
}

AND

ROBERT F. BACK.

(From the Medical Out-Patient Department, Royal Prince Alfred Hospital, Sydney.)

In view of the uncertainty of the usual results of specific desensitization methods in allergic types of bronchial asthma, this enquiry was undertaken to determine whether the claims made in some quarters for the value of a course of injections of the patient's own blood in the relief of this distressing malady were justified or not. The reactions of the skin to foreign proteins of all classes of antigens, particularly pollens, dusts, foods, bacteria, orris root, etc., have been used in the past. Desensitization seriatim or by a mixture of the apparently offending substances has usually proved fruitless in our department. The skin sensitivity to the scratch method has varied greatly in the same individual tested at intervals. It appears to be a practical impossibility in hospital practice to test each child for every possible antigen to which he is exposed. The method of autohaemotherapy seemed a simple and easily-applied type of parenteral injection therapy which on grounds of economy should appeal to the most conscientious hospital board.

The series of asthmatic patients reported as treated by this method is small. The procedure is by no means new, as Kahn and Emsheimer ${ }^{1}$ in 1916 obtained good results in six successive patients; Henske ${ }^{2}$ subsequently was satisfied with the improvement in nine of sixteen patients and Carranza and Orgaz $^{3}$ reported ten out of thirteen patients to be ' much improved.' Achard and Flandin ${ }^{4}$ used $0.5-2$ c.c. doses every $12-24$ hours, with better results in other allergic disorders than in asthma.

\section{Present investigation.}

Twenty-four patients (sixteen boys, eight girls) were selected for trial by this method. Before any injections were given the history of each child's illness was examined in detail and, unless this was thoroughly characteristic of an allergic form of bronchial asthma, the child was omitted from the series. A careful physical examination was supplemented by a otorhinologist's opinion upon the condition of the nose, throat and accessory nasal sinuses. An x-ray examination of the lungs and nasal sinuses and a fractional testmeal were carried out and finally the child's skin sensitivity was investigated 
by the scratch method to the proteins of the ordinary foods, bedding materials, house dust, pollens, orris root, linseed and, frequently, to bacteria. If surgical attention was required for the nose or throat, this was usually provided before the first injection was made. Advice as to diet, elimination of allergens from the environment, and other forms of treatment or desensitization were deliberately withheld. Some children were taking dilute hydrochloric acid with their meals; this was suspended. All were supplied with a stock mixture containing ammonia, lobelia, iodide of potassium and senega for varying periods before the haemotherapy began.

Method.-A constant dosage of 10 c.c. of the child's own blood was withdrawn from the median basilic vein and injected forthwith into the buttock, without admixture with citrate and without any attempt to separate either plasma or serum. Five such transferences of blood were made in each patient at weekly intervals. A careful record was kept of the number and severity of the attacks during the course itself and for a period varying from three to twelve months afterwards. In assessing the results, the mother's description of the number and nature of the attacks was the only criterion. Similarly, a comparison between the description of the seizures before and after treatment could only be drawn from the parent's statements. Changes in the child's general appearance and body-weight were noted, since in almost all instances the patients were seen again at approximately monthly to threemonthly intervals, even if their malady had abated sufficiently to render less visits to the hospital necessary. Skin sensitivity was re-tested in instances in which ' great improvement' was noted.

Results.-The difficulties of assessing the results of any treatment of asthma are notorious, especially when the series is small. Indeed, it is quite impossible to be at all dogmatic. The ever-changing allergic reaction in predisposed individuals provides the main stumbling block. No satisfactory measure of this state has yet been devised. Skin sensitivity is on the whole an unreliable index. Besides this quantitative variation in the intensity of the reaction as a whole, individual qualitative changes occur, whereby an allergic individual may alter the nature of his allergens during a period of medical observation such as that in the present series. In asthmatic children, who have usually a highly nervous make-up, the question of the psychological effect of these injections is uncertain. A conscious or unconscious determination not to require a repetition of the experience of sharp needles or Ryle's tube, may react favourably upon the severity of the disease in the post-injection period. As far as knowledge and experience is informative of other factors which may disturb the rhythm of the asthmatic state, a critical endeavour has been made to recognize conditions which could so vitiate the figures obtained. It has been observed that following the operation of tonsillectomy in childhood, there is often a relative or absolute remission from asthma, which may endure as long as twelve months. A change in address or other conditions of domestic environment has been enquired for. The presence of nasal or paranasal sepsis or of advanced 
changes in the anatomy and pathology of the chest have meant either the elimination of a potential patient from the series, or if included, the results are assessed separately (class II). Furthermore, when a long interval has previously been admitted to exist between attacks, the patient has also been considered separately from the severer sufferers as follows:-

Class I. Patients in whom, as far as could be discovered, the injections have had a fair and unhindered opportunity of affecting the course of the disorder : 14 children.

Class II. Patients in whom other factors noted above have been present which may possibly have had an independent effect on the reading of the results: 10 children.

The results obtained in each class are described in terms of groups, as follows :-

Group I. No attacks of significance during at least six months.

Group II. Fewer and less severe attacks.

Grour III. Attacks as frequent during period of observation, but less severe.

Group IV. No improvement noted.

$\begin{array}{lllccccc} & & & \text { Group I } & \text { Group II } & \text { Group III } & \text { Group IV } & \text { Total } \\ \text { Class I } & \ldots & \ldots & 6 & 4 & 4 & 0 & 14 \\ \text { Class II } & \ldots & \ldots & 3 & 4 & 3 & 0 & 10 \\ \text { Total } & \ldots & \ldots & 9 & 8 & 7 & 0 & 24\end{array}$

\section{Discussion.}

The figures are too small to convert into percentages; but it is obvious at a glance that in each child treated something has occurred for the better, and it can be recorded that each patient, as will be seen from the protocols appended, is satisfied that the procedure has been to some degree at least beneficial. In nearly one half of those patients constituting group I a definite relief has followed. It is not to be expected, ab initio, that a procedure such as this is in any way of permanent benefit, but the palliative effect seems to last an average period of about six months. Cases 1, 2, 3 and 7 have been observed for twelve months, and in the first three of these the benefit has been maintained throughout this period.

Apart from psychological effects, it is interesting but difficult to conjecture the manner by which any benefit may accrue. There is no general reaction observable as with protein shock therapy. It is possible that tissue digestion of the blood protein releases peptone which is gradually absorbed as such. Very small amounts of Oriel's proteose may be present in minute desensitizing doses in the injected blood. It was intended to compare the results of the injection of separated washed corpuscles and plasma respectively, but this would require a further two years' observation, and more juvenile asthmatics than reach one medical out-patient clinic of a 
general hospital. This small investigation was begun with an open mind. In assessing the results the authors have been deliberately critical concerning this application of the strange procedure of autohaemotherapy, which has given definitely favourable results. While the relief given may be quite temporary, by some means or other it gives the child a literal ' breathing space' during which it can develop physically and intellectually and so catch up with its more fortunate playmates.

\section{Subsidiary investigations.}

(a) Skin TESTs.-Effect of injections upon skin sensitivity: relation of our results thereto.

\begin{tabular}{|c|c|c|c|c|}
\hline & & & Group I & $\begin{array}{l}\text { ASSES I AND II } \\
\text { GROUPS II, III AND IV }\end{array}$ \\
\hline Skin teStS positive & $\ldots$ & $\ldots$ & 9 & 6 \\
\hline SkIN TESTS NEGATIVE & $\ldots$ & $\ldots$ & 2 & 6 \\
\hline
\end{tabular}

The majority (fifteen out of twenty-four) of the patients demonstrated skin sensitivity to the common allergens. Nine (three-fifths) of these patients appeared to respond well to autohaemotherapy. Eight patients were negative to the scratch technique. Of these only two (one-quarter) responded as well as any of the nine children whose skins reacted as above.

(b) AGe and heredity.-The influence of age, age of onset and heredity was also investigated, as follows:-

\begin{tabular}{llcccccc} 
& \multicolumn{3}{c}{ Class I } & \multicolumn{2}{c}{ Class II } & Total \\
Under 10 & years & Gre I, II & Gr. III, IV & Grp. I, II & Grp. III, IV & \\
10 & 6 & 3 & 1 & 0 & 10 \\
years and over & 3 & 3 & 4 & 4 & 14
\end{tabular}

The numbers are too small to justify any final conclusions, but the impression is left that children under ten years of age respond better than those in later childhood. It is probable that this applies to other forms of treatment in juvenile asthma where the history is necessarily of shorter duration.

Only eight out of twenty-four asthmatic children were of allergic stock. The average age of onset of asthma in these eight children was 5.2 years, as compared with the remainder of the series whose average age of onset was 4.4 years. As far as this goes, it gives no support to the belief that a history of allergic heredity determines an earlier onset in childhood.

\section{Conclusions.}

1. Twenty-four asthmatic children received five injections each of 10 c.c. of their own blood. Observations over a period of six to twelve months afterwards indicated that in approximately three-quarters the frequency of attack was appreciably reduced, while in every instance some alleviation in severity was manifest. 
2. Subsidiary observations were:-

(a) That the operation of tonsillectomy and removal of adenoids in childhood is alone capable of causing a remission of seizures for as long as twelve months.

(b) That the average age of onset of the asthma in this series was no earlier in children whose blood relatives had suffered similarly.

(c) That the results of haemotherapy seemed more favourable in children under the age of ten years.

(d) That the presence of skin sensitivity to the scratch method favoured better results from autohaemotherapy than its absence.

\section{Case records.}

Case 1.-T.W., a boy aged 7, had asthma for six years. His uncle and aunt were similarly affected. He showed some chest deformity and positive skin tests to pollens, dust, feathers, kapok and animal hair. Before treatment he was having attacks about every two months, lasting two days. After treatment his mother stated that ' he had improved beyond all expectation' and that though he had occasioned colds, no attacks were observed for the first six months. Two slight attacks occurred after twelve months. (Class I, group I.)

Case 2.-E. B., a boy aged 5, had asthma for eighteen months. His grandfather and uncle were also asthmatic. He was a thin child with bronchitic signs and showed positive skin tests to dust and kapok. Before treatment he was having attacks about every month, which were severe and lasted for twenty-four hours. After treatment he had only three slight attacks in ten months. (Class I, group II.)

Case 3.-A. P., a girl of 12, had asthma for ten years. Attacks occurred most generally in the stone-fruit season, when they occurred almost weekly and lasted for two days. Otorhinolaryngological examination revealed small polypi (right antrum wash-out showed pus). Skin tests for house dust and kapok were positive. After treatment she had one severe attack six months later, but no further attacks. The mother said that it was her ' first summer without attacks since she was a young baby.' (Class II (doubtful: a. seasonal, b. antrum), group I.)

Case 4.-W. R., a boy of 6, had asthma for four years. He was a thin child with bronchitic signs and had attacks about every three weeks (though sometimes weekly) which commenced by sneezing and lasted twenty-four hours. The attacks were generally provoked by cold winds. X-ray examination of his chest suggested the possibility of bronchiectasis at the right base. Skin tests were negative. For two months after treatment the child was free from attacks and the mother stated that it was the ' longest spell for three years.' After six months there had been some sneezing attacks, but no further asthma. The first mild asthmatic attack occurred nine months after treatment. (Class I, group II.) 
Case 5.-J. B., a boy of $12 \frac{1}{2}$, had asthma for nine-and-a-half years. A maternal aunt and a paternal uncle were similarly affected. The tonsils were enlarged, breath sounds harsh, and there was evidence of antritis. ('T'onsils and adenoids were removed one month before the injections were begun.) Heavy meals, especially pastry, seemed to cause attacks and skin tests to house dust and plantain were positive. Attacks occurred every two weeks and lasted from two to three days. After treatment there was one mild attack in five months, then the most severe attack for two years. The child was still sniffling and had some nasal discharge. A year later there were four more severe attacks within eight months. The mother stated that the attacks now ' lasted only one day instead of two or three days.' (Class I, group II or III.)

Case 6.-F. O., a boy of 9, had asthma for four years. The right antrum was opaque. Skin tests were negative. Before treatment there were two attacks in six months, the attacks lasting for two days. The child seemed to have a slight attack after each injection. After treatment he had slight attacks about every two months for six to eight months, then the attacks became as bad as before. The course of treatment was repeated. (Class II, group III or IV.)

Case 7.-A. R., a boy of 10, had asthma for nine months, which seemed to be caused by cold, dry winds. There were marked chronic bronchitic changes and the tonsils were infected): (they were removed two months after the injections were given). Attacks before treatment occurred about every two months and lasted a week. Dating from the first injection, there were no attacks for six months. Then there was slight wheezing and two bad attacks and four slight attacks in the twelve months following the last injection. (Class I, group II or III.)

Case 8.-A. M., a boy of 11, had asthma for seven-and-a-half years, the attacks occurring mostly in summer. He showed some permanent changes in the chest, increased hilar markings and positive skin tests to kentucky blue and dust. After treatment there were six attacks in three months; the attacks were less severe and frequent than before. A second course of treatment was given at the end of four months; there was one slight attack in the following two months. In this instance the injections were given early in the summer. (Class I, group II.)

Case 9.-N.W., a boy of 8 , had asthma for seven years, probably caused by grasses and kapok. Skin tests were positive to kapok, linseed, kentucky blue and dust. Examination of the chest revealed well-marked chronic bronchitis. Before treatment attacks occurred weekly and lasted for one or two days. Following treatment the child had only three slight attacks in seven months; the mother stated that he was 'greatly improved.' (Class I, group I.)

Case 10.-H. H., a boy of 9, had asthma for five years, the attacks being worse in winter. The breath sounds were harsh and there were small tonsillar tags. Skin tests were positive for house dust, horse dandar, kapok, plantain and cape weed. Before treatment attacks lasting two days occurred about every two weeks. After treatment there were only four slight attacks in seven months. The mother stated that the child was ' 90 per cent. improved.' (Class I, group I.) 
Case 11.-E. B., a girl of 9, had asthma for twelve months. She seemed to be affected by dusty rooms and the skin test to house dust was positive. Examination revealed chronic bronchitis and enlarged hilar glands. Tonsils and glands were enlarged: (tonsillectomy was performed). The attacks occurred every two months and lasted for two days. During the course of treatment the child had six very slight attacks; in the seven months following treatment there were twelve slight attacks. The mother reported that her general health was greatly improved. (Class I, group II.)

Case 12.-A.H., a boy of $15 \frac{1}{2}$, had asthma for ten years; the attacks occurred almost weekly and lasted for two days. There was well-marked chronic bronchitis, but no other signs. During the treatment three attacks occurred, hut there were no attacks in the two months following. The boy gained $11 \mathrm{lb}$. in weight. (Class I, group I.)

Case 13.-M. H., a girl of 9, had asthma for four years. An uncle was similarly affected. The attacks occurred in spring or early summer and skin tests to kentucky blue and house dust were positive. Examination of the chest showed an increase in hilar shadows towards the right upper lobe. Attacks occurred four times a year and lasted for two days. After treatment there were no attacks for four months (autumn); there was one attack in the next two months. The mother said that the girl's health had greatly improved since the injections. (Class II, group I.)

Case 14.-E. T., a girl of 14, had asthma for eighteen months. Her father was similarly affected and also one sister, mentioned in this series. Her tonsils were unhealthy and x-ray examination of the chest showed slight fibrosis. The attacks were worse in winter, but skin tests were negative. After treatment there were no attacks for six months. (Class I, group I.)

Case 15.-N.R., a girl of 16, had asthma for ten years. There were some chest changes and the left antrum was opaque. Skin tests for horse dander and house dust were positive. Before treatment attacks had occurred nightly for two months. In the six months following the commencement of treatment there were only two atiacks. The mother stated that the girl had gained considerably in weight. (Class I, group I.)

Case 16.-B. B., a boy of 12, had asthma all his life. He showed slight increase in lung markings towards the bases and positive skin tests to house dust and flock. Attacks occurred monthly and lasted a week. There were no severe attacks in the six months following the beginning of treatment. (Class I, group I.)

Case 17.-A. C., a boy of 10, had asthma for six years. There was well-marked chronic bronchitis and the nasal septum was enlarged. The skin test for plantain was positive. Before treatment attacks occurred about every two weeks and lasted for two days. There were three severe attacks during the two months of treatment. (Class I, group III.)

Case 18.-D. E., a girl of 6, had asthma for four years. X-ray examination of the chest revealed chronic bronchitic changes at both bases. Attacks had been less severe in the twelve months before treatment. There were frequent slight attacks during treatment and for the month following. Four months after 
injections mother states 'better than ever been.' (Class I, group II.)

Case 19.-B. W., a girl of 16, had asthma for four years. She was thin and nervous and suffered from middle-ear deafness. Before treatment attacks occurred almost nightly. In the two-and-ahalf months following beginning of treatment there were two very mild attacks; in the next six months there were six attacks; then the attacks became as bad as before. Class I, group III.)

Case 20.-M. B., a boy of $7 \frac{1}{2}$, had asthma for three years. His brother and maternal grandfather were similarly affected. He had frequent colds and showed positive skin tests to kentucky blue and plantain. Attacks occurred every three or four weeks. There were no attacks during the month following the commencement of treatment. (Class I, group ?.)

Case 21.-W. B., a boy of 9, had asthma for three years. Members of his mother's family were similarly affected. The attacks were worse in summer and the skin test for house dust was positive. Examination revealed minor bronchitic changes and mucosal thickening of the right antrum. Attacks occurred at less than monthly intervals and lasted from three to seven days. In the two months following the first injection there were five shorter attacks; then for two weeks there were no attacks; after which the attacks became as frequent and severe as before. The mother said that there was 'probably a little improvement.' (Class I, group III.)

Case 22.-G. B., a girl of 9, had asthma for eight years. She showed marked bronchitis, possibly bronchiectasis, and positive skin tests for kapok, plantain, horse dander and duck feathers. Before treatment the attacks occurred every three months or less and lasted for three days. There were three mild attacks in the two months after the first injection. The mother reported that she was ' a lot better' and that the attacks were not so frequent or severe. (Class I, group II or III.)

Case 23.-A.S., a boy of 8 , had had three or four bad attacks only. His grandmother was similarly affected. He showed slight chronic bronchitis and skin tests were negative. The attacks had been some weeks apart and lasted for three days. There were no attacks in the month following the first injection. (Class I, group ?.)

Case 24.-T. E., a boy of 14, had asthma for seven years, the attacks being worse in early spring. The skin test for kentucky blue was positive. Attacks occurred about six-monthly and lasted only one day, but were severe. There was no attack in the six months, including the spring, following the first injection. (Class I, group I.)

\section{REFERENCES.}

1, 2, 3, 4. Quoted by Bray, G. W., Rec. Advances in Allergy, London, 1931.

5. Durie, E. B., Kempson Maddox, Med. J. Australia, Sydney (in the Press). 